\title{
Estabilidade de clusters Pd-Cu com poucos átomos
}

\author{
Guilherme S. Francisco \\ Departamento de Física - Universidade Federal de Goiás - Campus Catalão, \\ Catalão, GO, Brazil \\ guilhermesousa_f@hotmail.com \\ N. L. Moreira \\ Departamento de Física - Universidade Federal de Goiás - Campus Catalão, \\ Catalão, GO, Brazil \\ nilton.lmoreira@gmail.com
}

\begin{abstract}
In this work we presents a study of the stability of palladium-copper bimetallic clusters with few atoms using Density Functional theory. The biding energy and dissociation energy was calculated to give information about the stability of the clusters. The results have shown the dependence of the stability of the clusters with its copper content.
\end{abstract}

Keywords - Bimetalic clusters, biding energy, dissociation energy, stability.

Resumo Neste trabalho apresentamos um estudo de clusters bimetálicos de paládio( $\mathrm{Pd})$ cobre $(\mathrm{Cu})$ com poucos átomos por Teoria do Funcional da Densidade. Calculamos a energia de ligação e energia de dissociação para obter informações sobre a estabilidade dos clusters. Os resultados mostram a dependência da estabilidade com o número de átomos de cobre nos clusters.

Palavras-chave - Clusters bimetálicos, energia de ligação, energia de dissociação, estabilidade.

\section{INTRODUÇÃO}

Atualmente clusters de metais de transição tem atraído muita atenção tanto do ponto de vista fundamental quanto de aplicações tecnológicas por conta das propriedades catalíticas únicas desses materiais. O paládio é um metal de transição que está presente na indústria desde a manufatura de dispositivos para celulares, computadores até a indústria aeroespacial. Aplicações na indústria automobilística também são importantes, por exemplo, clusters de $\mathrm{Pd}$, como também $\mathrm{Pt}$ e Rh, são usados para reduzir a emissão de poluentes tóxicos como $\mathrm{CO}$ e $\mathrm{NO}$ produzidos na queima de combustíveis (Kalita, 2007). Estudo de clusters $\mathrm{Pd}$ tem mostrado propriedades ferromagnéticas(Kumar, 2002), abrindo possibilidade para novas aplicações como armazenamento em meios magnéticos.

Várias propriedades dos clusters de Pd puros já foram estudadas tais como a transição isolante metal usando o método Tight-binding (Aguilera-Granja, 2007) e Teoria do funcional da densidade (DFT-Density functional theory) (Aguilera-Granja, 2007; Kumar, 2002). Propriedades geométricas e eletrônicas de clusters neutros, aniônicos e catiônicos por DFT (Kalita, 2007). Efeitos da estrutura sobre as propriedades energética, eletrônica e magnéticas de nanopartículas de paládio (Moseler, 2001; Zhang, 2003). Estudo de propriedades de clusters bimetálicos também são encontrados como AuPd (Liu, 2012) e PdCu (Efremenko, 2005) por DFT.

Neste trabalho apresentamos um estudo sobre a estabilidade de clusters bimetálicos de $\mathrm{Pd}_{n} \mathrm{Cu}_{m}$ com $m+n=6$. Informações sobre a estabilidade dos clusters podem ser obtidas através da energia de ligação (BE), energia de dissociação $(\Delta E)$. Este artigo está organizado da seguinte forma: Na seção 2 apresentamos os detalhes computacionais e metodologia utilizadas. Na seção 3 apresentamos nossos resultados e discussões e finalmente, na seção 4 apresentamos nossas conclusões.

\section{DETALHES COMPUTACIONAIS}

As estruturas geométricas e eletrônicas dos clusters $\mathrm{PdCu}$ com poucos átomos foram obtidas dentro da aproximação GGA usando um funcional de troca e correlação híbrido B3LYP como implementado no pacote GAUSSIAN98. Utilizamos a base LAN08(f) como descrito em (Hay, 1984-1; Hay, 1984-2; Willard, 1984), com pseudo potencial incluso, e que levam em conta efeitos relativísticos que influenciam os 
elétrons das camadas mais externas. As estruturas foram obtidas a partir de uma estrutura $\mathrm{Pd}_{2} \mathrm{Cu}_{4}$ por meio de substituição sistemática de átomos de $\mathrm{Pd}$ e $\mathrm{Cu}$ e então otimizadas usando DFT. Primeiramente, clusters puros $\mathrm{Cu}_{6}$ foram obtidos substituindo os átomos de $\mathrm{Pd}$ por átomos de $\mathrm{Cu}$ em todas as combinações de posições possíveis. De forma semelhante, clusters puros $\mathrm{Pd}_{6}$ foram obtidos por substituição dos átomos de $\mathrm{Cu}$ por átomos $\mathrm{Pd}$ considerando todas as combinações de posições possíveis, preenchendo todo o triângulo como mostrado na figura 1. Consideramos aqui as estruturas com menores energias.

\section{RESULTADOS}

Nesta seção apresentamos as estruturas geométricas e as diferenças de energias calculadas para os clusters $\mathrm{Pd}_{n} \mathrm{Cu}_{m}$. A figura III mostra esquematicamente todas as famílias de clusters calculadas neste trabalho. Calculamos a energia de ligação e comparamos com resultados teóricos e experimentais (quando houver) da literatura e apresentamos a energia de dissociação como mostrados a seguir.

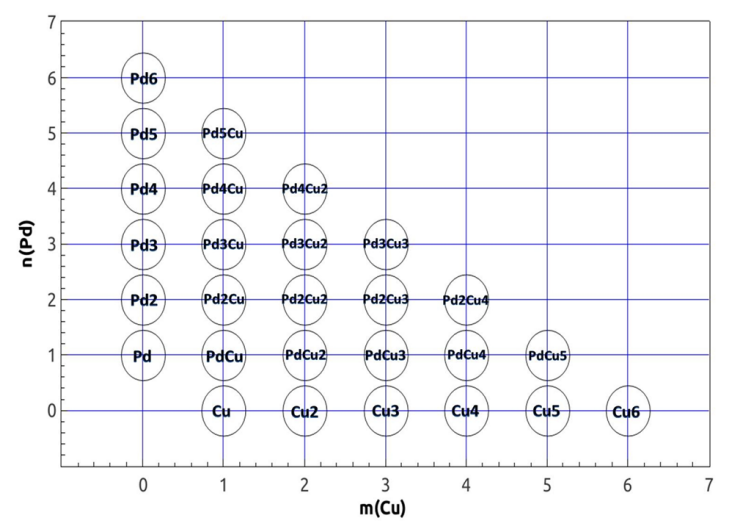

FIG. 1: Famílias de clusters calculados.

A figura 2 mostra as estruturas geométricas como mostradas esquematicamente na figura III. A linha horizontal inferior mostra os cluster $\mathrm{Cu}$ puros. A primeira coluna a esquerda mostra clusters de Pd puros. O cruzamento das várias linhas e colunas formam os clusters mistos com $m+n=6$. Os resultados mostram boa concordância geral com a literatura, contudo, diferenças pontuais foram encontradas. Para cluster $\mathrm{Cu}_{2}$ encontramos distância interatômica $d_{c_{2}}=0.035 \AA$ maior que o valor experimental que é $2.223 \AA$ [Rohlfing,(1095)].
A figura 2 mostra estrutura plana para ambos, $\mathrm{Pd}_{4}$ e $\mathrm{Cu}_{4}$, em contraste com as estruturas tridimensionais apresentadas. A estrutura tetraédrica $\mathrm{Pd}_{4}$ tem energia $0.011 \mathrm{eV}$ maior que a energia da estrutura planar mostrada na figura 3. As estruturas mistas para $m+n=4$ são tetraédricas com ligações $\mathrm{Pd}-\mathrm{Cu}$ menores que as ligações Pd-Pd. Para $m+n=5$ todos os clusters apresentam estruturas na forma bipiramidal. Para $m+n=6$ os cluster apresentam estrutura na forma de octoedros para $\mathrm{Pd}_{6}$, $\mathrm{Pd}_{5} \mathrm{Cu}, \mathrm{Pd}_{4} \mathrm{Cu}_{2}$ e $\mathrm{PdCu}_{5}$. Os demais apresentam forma de bipirâmides trigonais, também em concordância com resultados anteriores. A despeito da impossibilidade de se comparar as energias totais, as diferenças de energia podem ser comparadas como mostradas a seguir.

\section{A. Energia de Ligação}

A energia de ligação é calculada como

$$
B E=E_{P d_{n} C u_{m}}-n E_{P d}-m E_{C u}
$$

A figura 3 mostra as energias de ligação e sua dependência com a composição dos cluters. Os

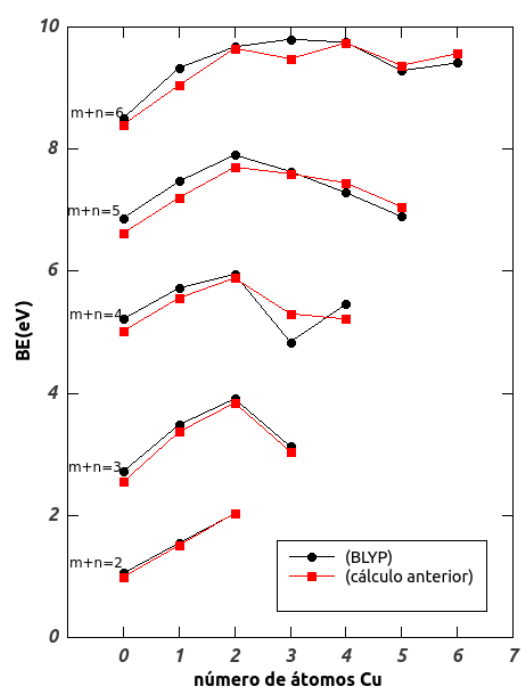

FIG. 3: Energias de ligação para os clusters Pd$\mathrm{Cu}$. Círculos cheios (pretos) nossos resultados e quadrados cheios (vermelhos) os da referência (Efremenko,2005).

círculos cheios são os nossos valores. Os quadrados cheios são os da referência (Efremenko, 2005). Os resultados mostram que para sistemas monometálicos, clusters de $\mathrm{Cu}$ são mais estáveis que os clusters de Pd. A 


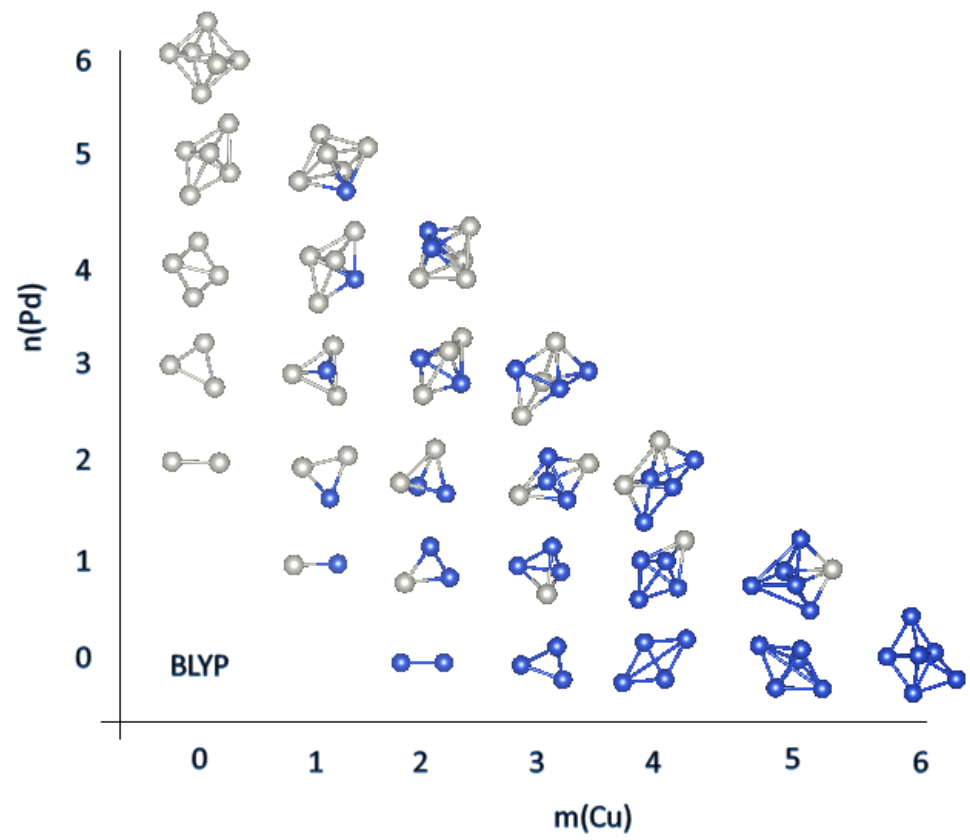

FIG. 2: Estruturas geométricas obtidas. No eixo horizontal apresentamos composição em átomos de $\mathrm{Cu}$. No eixo vertical apresentamos composição em átomos de Pd.

figura mostra ainda a boa concordância qualitativa encontrada para $m+n \leq 6$ onde maior energia de ligação implica em maior estabilidade para clusters $\mathrm{Pd}_{n} \mathrm{Cu}_{2}$ com $n+2 \leq 6$ a despeito das diferenças na base e no pseudo potencial utilizados nos nossos cálculos. Para $n+m=6$ nossos resultados mostram maior energia de ligação para $\mathrm{Pd}_{3} \mathrm{Cu}_{3}$.

Para que haja interação é necessário que os átomos passem de um estado fundamental singleto, com uma configuração eletrônica $4 d^{10}$, $5 s^{0}$, para tripleto, com configuração $4 d^{9}, 5 s^{1}$ para a formação de nanopartículas, logo, o estado fundamental dos clusters paládio puro são tripletos. Por sua vez, os átomos de $\mathrm{Cu}$ possuem configuração eletrônica $3 d^{9}, 4 s^{2}$. Essa configuração favorece a formação de ligações, explicando a maior estabilidade dos clusters $\mathrm{Cu}_{m}$. Para sistemas bimetálicos a interação dos orbitais $4 d$ e $5 s$ do $\mathrm{Pd}$ com os orbitais com $4 s$ do $\mathrm{Cu}$ tem o mesmo efeito de excitação do $\mathrm{Pd}$ conferindo maior estabilidade para os clusters bimetálicos mantendo o estado fundamental singleto.

\section{B. Energia de Dissociação}

Informações adicionais sobre a estabilidade dos clusters, ou sua estabilidade em relação aos seus vizinhos, podem ser obtidas calculando a energia de dissociação dos clusters. Maior energia necessária para remover um átomo do cluster implica em maior estabilidade. A equação 2 fornece a energia para dissociação de um átomo $\mathrm{Pd}$,

$$
\Delta E_{n}=E_{P d_{n-1} C u_{m}}+E_{P d}-E_{P d_{n} C u_{m}}
$$

A equação 3 , por sua vez, mostra a energia de dissociação de um átomo de $\mathrm{Cu}$ dos clusters,

$$
\Delta E_{n}=E_{P d_{n} C u_{m-1}}+E_{C u}-E_{P d_{n} C u_{m}}
$$

A figura 4 mostra os nossos resultados para as energias de dissociação calculadas a partir da equação 2. Mantendo o número de átomos de $\mathrm{Cu}$ fixo, as curvas mostram a evidente dependência da energia de dissociação com a composição de átomos de $\mathrm{Cu}$ nos clusters mostrando que a presença deste metal nos clusters bimetálicos determinam a estabilidade das 
nanopartículas. A figura 5 mostra a energias de dissociação calculadas a partir da equação 3 , mantendo-se o número de átomos de $\mathrm{Pd}$ fixos. Um aspecto adicional deve ser notado, para um mesmo número de átomos de $\mathrm{Pd}$ no cluster, maior estabilidade está associada à números pares de átomos de $\mathrm{Cu}$.

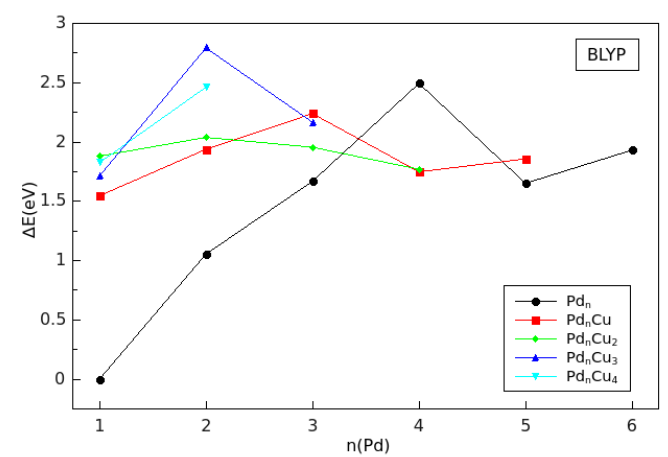

FIG. 4: Energia de dissociação de um átomo de $\mathrm{Pd}$ dos clusters $\mathrm{Pd}-\mathrm{Cu}$.

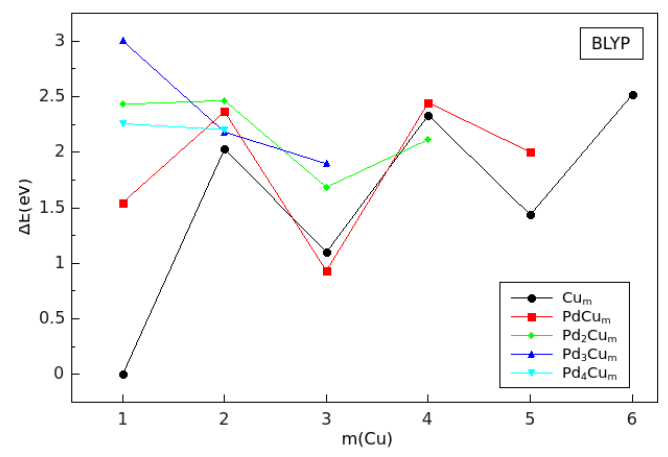

FIG. 5: Energia de dissociação de um átomo de $\mathrm{Cu}$ dos clusters $\mathrm{Pd}-\mathrm{Cu}$.

\section{CONCLUSÃO}

Apresentamos neste trabalho um estudo sobre clusters bimetálicos para determinação de suas propriedades geométricas e eletrônicas (que determinam sua estabilidade) usando DFT. Obtemos as estruturas geométricas para todas as combinações de $m$ e $n$ nos clusters $\mathrm{Pd}_{n} \mathrm{Cu}_{m} \operatorname{com} m+n \leq 6$ dentro da aproximação GGA usando o funcional B3LYP. Calculamos as energias de ligação e dissociação.

Os clusters puros de $\mathrm{Cu}_{m}$ mostram maior estabilidade que os clusters de $\mathrm{Pd}_{n}$ puros. Para os clusters mistos $\mathrm{Pd}_{n} \mathrm{Cu}_{m}$, maior energia de ligação (estabilidade) é observada para $m=2$ com $n+2<6$.

Para obter informações sobre a estabilidade relativa dos clusters bimetálicos, calculamos a energia de dissociação para esse sistema, algo que ainda não se encontra na literatura. No cálculo da energia de dissociação confirmou novamente que a estabilidade de um cluster está relacionada ao número de átomos $\mathrm{Cu}$ presentes nos clusters.

\section{AGRADECIMENTOS}

Agradecimento especial ao Professor Antônio Eduardo da Hora Machado por gentilmente ter gerado as estruturas iniciais utilizadas neste trabalho.
Aguilera-Granja, F., Vega, A., Rogan, J., García, G.; (2007). Metallic behavior of $\mathrm{Pd}$ atomic clusters. Nanotechnology, 18, pp 365706-365710.

Efremenko, I., Sheintuch. M., (2005), DFT study of small bimetallic palladium-copper clusters. Chemical Physics Letters, 401, pp 232-240.

Hay, P J., Wadt, W. R., (1984), Ab Initio effective core potentials for molecular calculations. Potentials for the transition metal atoms Sc to $\mathrm{Hg}$. The Journal of Chemical Physics, 82, pp 270-283.

Hay, P J., Wadt, W. R., (1984), Ab ini- tio effective core potentials for molecular calculations. Potentials for $\mathrm{K}$ to $\mathrm{Au}$ including the outermost core orbitals. The Journal of Chemical Physics, 82, pp 299-310.

Kalita, B., Deka, R. C., (2007). Stability of small $\mathrm{Pd}_{n}(n=1-7)$ clusters on the basis of structural and eletronic properties: A density functional approach. The Journal of Chemical Physics, 127, pp 244306-244315.

Kumar, V., Kawazoe, Y., (2002). Icosahedral growth, magnetic behavior, and adsorbateinduced metal-nometal transition in palladium clusters. Phys. Rev. B 66, pp 144413-144323.

Liu, Xuenjing; Tian, Doongxu; Meng, 
Changgong (2012). DFT study on stability and structure of bimetallic $\mathrm{Au}_{m} \mathrm{Pd}_{n}(N=38,55,79$, $N=m+n, m / n \approx 2: 1$ e $5: 1$ ) clusters. Computational and Theoretical Chemistry, 999, pp 246-250.

Moseler, M., Häkkinen, H., Barnet, R. N., Lamdman U., (2000). Structure and Magnetism of neutral and anionic palladium clusters. Phys. Rev. Let. 86, pp 2545-2548.

Rohlfing, E. A.,Valentini J. J.,(1985), UV laser excited fluorescence spectroscopy of the jet-cooled copper dimer. The Journal of
Chemical Physics 84, pp 6560-6566.

Willard A. Wadt and P. Jeffrey Hay, (1984),Ab Initio effective core potentials for molecular calculations. Potentials for main group elements $\mathrm{Na}$ to $\mathrm{Bi}$. The Journal of Chemical Physics, 82 , pp 284-298.

Zhang, W., Ge, Qingfeng, Wang, L.,(2003), Structure effects on the energetic, eletronic, and magnetic properties of palladium nanoparticles. Journal of Chemical Physics, 118, pp 5793-5801. 